\title{
Effects of Shu Gan Jian Pi formula on rats with carbon tetrachloride-induced liver fibrosis using serum metabonomics based on gas chromatography-time of flight mass spectrometry
}

\author{
HUI JIANG ${ }^{1,2}$, XIU-JUAN QIN ${ }^{2}$, WEI-PING LI ${ }^{1}$, RONG MA ${ }^{3}$, TING WANG ${ }^{2}$ and ZHU-QING LI ${ }^{1}$ \\ ${ }^{1}$ Department of Pharmacy, College of Basic Medicine, Anhui Medical University, Hefei, Anhui 230031; \\ ${ }^{2}$ Department of Pharmacy, The First Affiliated Hospital of Anhui University of Chinese Medicine, \\ Hefei, Anhui 230032, P.R. China; ${ }^{3}$ Institute for Cardiovascular and Metabolic Diseases, \\ University of North Texas Health Sciences Center, Fort Worth, TX 76107, USA
}

Received February 16, 2016; Accepted February 20, 2017

DOI: $10.3892 / \mathrm{mmr} .2017 .7078$

\begin{abstract}
Liver fibrosis is a common stage in the majority of chronic liver diseases, regardless of the etiology, and its progression may lead to hepatic cirrhosis or hepatocellular carcinoma. Metabolomics, a powerful approach in systems biology, is a discipline used to qualitatively and quantitatively analyze the small molecule metabolites of cells at specific times and under certain conditions. The present study aimed to investigate serum metabolic changes following Shu Gan Jian Pi formula (SGJPF) treatment of carbon tetrachloride $\left(\mathrm{CCl}_{4}\right)$-induced liver fibrosis in rats using gas chromatography-time of flight mass spectrometry (GC-TOFMS). In addition, the potential mechanisms were explored. Rat liver fibrosis was induced by twice-weekly subcutaneous $\mathrm{CCl}_{4}$ injection for 12 continuous weeks. During the same period, the SGJPF group received $16.2 \mathrm{~g} / \mathrm{kg}$ body weight SGJPF, diluted in water, once a day for 12 weeks. Rats in the control and model groups received oral administration of the same volume of saline solution. Serum samples from the control, model and SGJPF groups were collected after 12 weeks of treatment, and metabolic profile alterations were analyzed by GC-TOF/MS. Metabolic
\end{abstract}

Correspondence to: Professor Wei-Ping Li, Department of Pharmacy, College of Basic Medicine, Anhui Medical University, 81 Meishan Road, Hefei, Anhui 230031, P.R. China

E-mail: lwp196061@126.com

Abbreviations: $\mathrm{CCl}_{4}$, carbon tetrachloride; GC-TOFMS, gas chromatography-time of flight mass spectrometry; PCA, principal component analysis; OPLS-DA, orthogonal projections to latent structures-discriminate analysis; ECM, extracellular matrix; HE, hematoxylin and eosin; VIP, variable importance projection; KEGG, Kyoto Encyclopedia of Genes and Genomes; HSCs, hepatic stellate cells; RAAS, renin-angiotensin-aldosterone system

Key words: liver fibrosis, Shu Gan Jian Pi formula, serum, metabonomics, gas chromatography-time of flight mass spectrometry profile analysis indicated that clustering differed between the three groups and the following 12 metabolites were detected in the serum of all three groups: Isoleucine; L-malic acid; D-erythro-sphingosine; putrescine; malonic acid; 3,6-anhydro-D-galactose, $\alpha$-ketoglutaric acid; ornithine; glucose; hippuric acid; tetrahydrocorticosterone; and fucose. The results demonstrated that SGJPF treatment mitigated the effects of $\mathrm{CCl}_{4}$-induced liver fibrosis on biomarker levels, thus indicating that SGJPF may have a therapeutic effect on $\mathrm{CCl}_{4}$-induced liver fibrosis in rats. The mechanism may involve the regulation of energy, amino acid, sphingolipid, cytochrome P450, glucose and water-electrolyte metabolism.

\section{Introduction}

Liver fibrosis is a key pathological process leading to the development of chronic liver disease and cirrhosis, which is characterized by extracellular matrix (ECM) and collagen accumulation arising from an imbalance between fiber hyperplasia and ECM degradation (1). Liver fibrosis is reversible, whereas cirrhosis is irreversible $(2,3)$. Various synthetic chemicals that cure liver fibrosis have been introduced; however, these treatments are not very effective and display various side effects. Therefore, there is an urgent need to develop novel treatments for liver fibrosis. In the search for novel therapeutic products for the treatment of liver fibrosis, medicinal plant research has contributed significantly by demonstrating the pharmacological effectiveness of different herbs or their prescriptions (4).

In China, liver fibrosis belongs to the "costalgia, accumulation and jaundice' traditional Chinese medicine (TCM) category (5). Shu Gan Jian Pi formula (SGJPF), also known as Ganle granula or Ganle infusion is a TCM formula, which has been generally recognized as a classic prescription and has a curative effect on liver fibrosis $(6,7)$. The preparation is composed of Coix lacryma-jobi L. (Semen Coicis; Jobstears Seed; Yiyiren), Astragalus membranaceus (Fisch.) Bunge. (Radix Astragali; Milkvetch Root; Huangqi), Bupleurum chinense DC. (Radix Bupleuri; Chinese Thorowax root; Chaihu), Paeonia lactiflora Pall. (Radix Paeoniae Alba; 
White Peony Root; Baishao), Atractylodes macrocephala Koidz. (Rhizoma Atractylodis Macrocephalae; White Atractylodes Rhizome; Baizhu), Smilax china L. (Poria; Chinaroot Greenbrier Rhizome; Fuling), Polyporus umbellatus (Pers.) Fries. (Polyphorus; Grifola Umbellate; Zhuling), Eupatorium japonicum Thunb. (Herba Lycopi; Shiny Bugleweed Herb; Zelan), Isatis tinctoria L. (Radix Isatidis; Indigowoad Root; Banlangen) and Glycyrrhiza uralensis Fisch. (Radix et Rhizoma Glycyrrhizae; Licorice; Gancao).

In our previous study, extraction and preparation methods of the final SGJPF product were investigated (8). It was demonstrated that the extraction methods were appropriate, simple and feasible, and quality control data were available, which provided a theoretical basis for the production of this product. It has also been indicated that SGJPF exerts protective effects against carbon tetrachloride $\left(\mathrm{CCl}_{4}\right)$-induced liver fibrosis in rats via the suppression of tissue inhibitor of metalloproteinases-1 and B-cell lymphoma 2-associated X protein expression, which may be one of its therapeutic mechanisms (9). At present, the majority of studies have focused on the molecular biological mechanism of SGJPF; however, the metabonomic mechanism underlying the protective effects of SGJPF against liver fibrosis remains to be discovered $(9,10)$. Metabonomics is defined as 'the quantitative measurement of the dynamic, multiparametric metabolic response of living systems to pathophysiologic stimuli or genetic modification' $(11,12)$. It is a novel technological platform that provides information from the whole organism. Therefore, it complies well with the holistic theory and systemic features underlying TCM. It has been applied to various domains to estimate the effect and elucidate the mechanism of TCM. It also identifies potential associations between metabolic profile changes and the physiological status of the biosystems $(13,14)$. Various analytical tools have previously been used to analyze metabonomics, including fourier transform infrared spectroscopy, capillary electrophoresis mass spectrometry, hydrogen-1 nuclear magnetic resonance, high-performance liquid chromatography mass spectrometry and gas chromatography-time of flight mass spectrometry (GC-TOFMS) (15). GC-TOFMS is a robust, unbiased analytical tool, characterized by high sensitivity, reproducibility, separation efficiency, simplicity and National Institute of Standards and Technology database (http://srdata.nist.gov/) accessibility in identifying and quantifying metabolites. GC-TOFMS is considered a powerful and useful tool for metabonomic analysis (16).

The present study identified serum metabolic profile changes associated with $\mathrm{CCl}_{4}$-induced liver fibrosis in rats based on GC-TOFMS with multivariate statistical techniques, including principal component analysis (PCA), partial least squares-discriminate analysis (PLS-DA) and orthogonal projections to latent structures-discriminate analysis (OPLS-DA), which were used to estimate the effects of intervention with $\mathrm{SGJPF}$ on $\mathrm{CCl}_{4}$-induced liver fibrosis (17). By analyzing the metabolic profile alterations, the present study identified the potential mechanisms through which SGJPF may exert protective effects against liver fibrosis.

\section{Materials and methods}

Experimental animals. The protocol was approved by the Committee of the Ethics of Animal Experiments of The First
Affiliated Hospital of Anhui University of Chinese Medicine (permit no. 2012AH-037-02; Hefei, China). All surgical procedures were performed under isoflurane anesthesia and all efforts were made to minimize suffering. Adult male, specific pathogen-free Sprague-Dawley rats (180-200 g; age, 11-12 weeks old; $n=15$ ) were purchased from the Laboratory Animal Center of Anhui Medical University (Hefei, China). All rats were housed in standard cages at a temperature of $20 \pm 5^{\circ} \mathrm{C}$ under a $12 \mathrm{~h}$ day/night cycle. The rats were freely supplied with standard animal food and water.

Experimental chemicals. SGJPF was obtained from The First Affiliated Hospital of Anhui University Of Chinese Medicine and $\mathrm{CCl}_{4}$ was obtained from Shantou Xilong Chemical Plant Co. Ltd. (Shantou, China). L-2-chlorophenylalanine, pyridine, isoflurane and olive oil (Shanghai HC Biotech Co., Ltd., Shanghai, China) were of analytical grade.

$\mathrm{CCl}_{4}$-induced liver fibrosis and treatment groups. After a feeding adaptation period of 2 weeks, fifteen rats were randomly divided into three groups: the control, model and SGJPF groups ( $n=5 /$ group). Liver fibrosis in the model and SGJPF groups was established by a single subcutaneous injection of $50 \% \mathrm{CCl}_{4}\left(1.0 \mathrm{ml} / \mathrm{kg} ; \mathrm{CCl}_{4}\right.$ diluted in olive oil, $\mathrm{v} / \mathrm{v}=1: 1)$ twice a week for 12 weeks. The control group was injected with olive oil only for 12 weeks. SGJPF $(16.2 \mathrm{~g} / \mathrm{kg})$ was administered to the SGJPF group via gavage daily during the 12-week period. The control and model rats received oral administration of the same volume of saline solution.

Sample collection. At the end of the experimental period, animals were anesthetized with isoflurane $(3 \mathrm{ml} / \mathrm{kg})$. Serum samples were collected from all rats from abdominal aorta blood after 12 weeks of $\mathrm{CCl}_{4}$ treatment, left for $2 \mathrm{~h}$ at room temperature and then centrifuged at 4,200 x g for $15 \mathrm{~min}$ at room temperature. All serum samples were stored at $-80^{\circ} \mathrm{C}$ until further use.

Histology. At 12 weeks, the rats were anesthetized by intraperitoneal injection of isoflurane $(3 \mathrm{ml} / \mathrm{kg})$. A portion of each rat liver from the right lobe was fixed in $10 \%$ neutral formalin for $24 \mathrm{~h}$ at room temperature, then embedded in paraffin, cut into 4- $\mu \mathrm{m}$ sections. Paraffin-embedded liver sections were deparaffinized with xylene and rehydrated using an ethanol gradient $(100-70 \% \mathrm{v} / \mathrm{v})$, then the sections were stained with $0.5 \%(\mathrm{w} / \mathrm{v})$ hematoxylin (5-10 $\mathrm{min})$ and eosin (1-2 min; HE staining) at room temperature for general observation or with Masson staining for the detection of collagen deposition (18). Sections were photographed on a Leica light microscope (Leica Microsystems GmbH, Wetzlar, Germany).

Serum sample preparation. Serum samples $(100 \mu \mathrm{l})$ were added to a solution containing $350 \mu \mathrm{l}$ methanol and chloroform (v/v; 3:1) and $50 \mu 1 \mathrm{~L}-2$-chlorophenylalanine; the samples were then mixed by vortexing and were centrifuged at $11,250 \mathrm{x}$ g for $10 \mathrm{~min}$ at $4^{\circ} \mathrm{C}$. The resulting supernatant $(0.35 \mathrm{ml})$ was transferred into a $2 \mathrm{ml}$ vial. Extracts were dried in a vacuum concentrator, and were then incubated with $80 \mu \mathrm{l}$ methoxylamine (Sigma-Aldrich; Merck KGaA, Darmstadt, Germany) in pyridine $(20 \mathrm{mg} / \mathrm{ml})$ for $2 \mathrm{~h}$ at $37^{\circ} \mathrm{C}$ in an oven. Subsequently, the extracts were incubated 
with $100 \mu \mathrm{l}$ bis- (trimethylsilyl) trifluoroacetamide (1\% trimethylchlorosilane, v/v; Regis Technologies. Inc., Chicago, IL, USA) for $1 \mathrm{~h}$ at $70^{\circ} \mathrm{C}$. The mixture was then allowed to cool to room temperature.

GC-TOFMS analysis. GC-TOFMS analysis was conducted using an Agilent 7890 gas chromatograph system (Agilent Technologies, Inc., Santa Clara, CA, USA) with a Pegasus 4D time-of-flight mass spectrometer (LECO Corporation, St. Joseph, MI, USA). The system used a DB-5MS capillary column coated with 5\% diphenyl cross-linked and 95\% dimethylpolysiloxane $(30 \times 250 \mu \mathrm{m}$ inner diameter; $0.25 \mu \mathrm{m}$ film thickness; J\&W Scientific, Inc., Folsom, CA, USA). A $1 \mu 1$ aliquot of the analyte was injected in splitless mode. Helium was used as the carrier gas, the front inlet purge flow was $3 \mathrm{ml} / \mathrm{min}$, and the gas flow rate through the column was $1 \mathrm{ml} / \mathrm{min}$. The original temperature was maintained at $80^{\circ} \mathrm{C}$ for $0.2 \mathrm{~min}$, which was raised to $180^{\circ} \mathrm{C}$ at a rate of $10^{\circ} \mathrm{C} / \mathrm{min}$ and subsequently to $240^{\circ} \mathrm{C}$ at a rate of $5^{\circ} \mathrm{C} / \mathrm{min}$, finally the temperature was increased to $290^{\circ} \mathrm{C}$ at a rate of $20^{\circ} \mathrm{C} / \mathrm{min}$. The injection temperature was $280^{\circ} \mathrm{C}$, transfer line and ion source temperatures were $270^{\circ} \mathrm{C}$ and $220^{\circ} \mathrm{C}$, respectively. The energy was $-70 \mathrm{eV}$ in electron impact mode. The mass spectrometry data were acquired in full-scan pattern with an $\mathrm{m} / \mathrm{z}$ range of 20-600 at a rate of 100 spectra per second following a solvent delay of $492 \mathrm{sec}$.

Statistical and data analysis. Chroma TOF4.3X software (LECO Corporation) and LECO-Fiehn Rtx 5 database (LECO Corporation) were used for data baseline filtering, raw peak exaction, peak alignment and identification, calibration of the baseline, deconvolution analysis and integration of the peak area. The peaks were normalized to the sum of spectrum prior to multivariate analyses. All raw data were analyzed by PCA, PLS-DA and OPLS-DA using SIMCA-P+11.5 software (MKS Data Analytics Solutions, Malmö, Sweden) after performing a unit variance procedure. To further identify potential biomarkers, commercial databases, including the Kyoto Encyclopedia of Genes and Genomes (KEGG; http://www. kegg.jp/kegg/) and PubChem Compound (https://pubchem. ncbi.nlm.nih.gov/), were used to search for metabolites. Then physiological, biochemical and pathological information was collected from CytoKEGG (Cytoscape 3.0.1 software; http://www.cytoscape.org/) to analyze the potential biomarkers involved in the metabolic pathways (19). Data were processed by SPSS 17.0 (SPSS, Inc., Chicago, IL, USA) and statistical analyses were performed using the one-way analysis of variance (ANOVA) among multiple groups followed by Fisher's least significant difference test. $\mathrm{P}<0.05$ was considered to indicate a statistically significant difference.

\section{Results}

Histopathological alterations in hepatic tissue. To assess histopathological alterations, HE and Masson staining was performed on liver tissues from all rats. Analysis of the control group demonstrated that the liver cell structure was clear, the liver cell nucleus was large and round, the nucleolus was obvious and the cytoplasm was abundant with minimal collagen deposition (Fig. 1A and B). Conversely, the model group exhibited increased fatty degeneration (arrow, a), inflammatory cell infiltration (arrow, b), a dissolved nucleus (arrow, c) and increased hyperplastic collagen fibers (arrow, d) compared with the control group (Fig. 1C and D). Following SGJPF intervention, there was less fatty degeneration, inflammatory cell infiltration, dissolved nuclei and collagen deposition when compared with the model group (Fig. 1E and F).

Total ion chromatogram of serum samples. Typical GC-TOFMS total ion current chromatograms of rat serum following 12 weeks of $\mathrm{CCl}_{4}$ treatment in the control, model and SGJPF groups are presented in Fig. 2. Based on the LECO-Fiehn Rtx 5 database, 469 metabolites were identified. After using Chroma TOF4.3X software to correct for blank values, eliminate noise and correct to an internal standard, 380 metabolites were identified. The $\mathrm{x}$-axis represents the time at which metabolites occurred, and each peak represents the relative metabolite expression (20).

$P C A$. To illustrate the general trend, PCA was performed to explain as much variation in as few components as possible. PCA displays the internal structure of datasets in an unbiased manner and decreases the dimensionality of the data (21). In the PCA score plot, each data point represents a rat sample following 12 weeks of $\mathrm{CCl}_{4}$ treatment, and the distance between points in the score plot is an indication of the similarity between samples. As presented in Fig. 3, the control, model and SGJPF groups were not completely separate; however, there were certain differences between the groups.

PLS-DA. To further detect differences between groups and identify potential biomarkers, supervised PLS-DA was performed. It is based on the leave-one-out cross-validation method (22). $\mathrm{R}^{2}$ is the fraction of the sum of squares explained by the model and represents model fitness. $\mathrm{Q}^{2}$ is the fraction of $\mathrm{Y}$ variation predicted by the $\mathrm{X}$ model in that component, which represents predictive ability and is used for specific components to judge the validity of the model (23). Following PLS-DA analysis, the control, model and SGJPF groups were completely separated. The $\mathrm{R}^{2} \mathrm{Y}$ was 0.934 and the $\mathrm{Q}^{2} \mathrm{Y}$ was 0.561 , which indicate that the model has a good fit and reliable predictive ability. After permutation testing, $\mathrm{R}^{2}$ and $\mathrm{Q}^{2}$ (predictive ability) were 0.725 and -0.22 , respectively, indicating that the model was reliable, and not over-fitted. The results are presented in Fig. 4.

OPLS-DA. The OPLS-DA score plot of the control, model and SGJPF groups indicated that the three groups were completely separated (Fig. 5A). Furthermore, SGJPF treatment affected the metabolism of rats with $\mathrm{CCl}_{4}$-induced liver fibrosis. The corresponding OPLS-DA loading plot (Fig. 5B) indicated that a metabolic substance manifested as one of the most influential variables according to its respective contribution to the discrimination (24). Each dot in the loading plot represents a metabolite. Dots near the center represent small differences in model classification, whereas dots far from the center were considered to have a higher contribution to the model classification. Red dots indicate the 12 potential biomarkers that were identified in this experiment. 

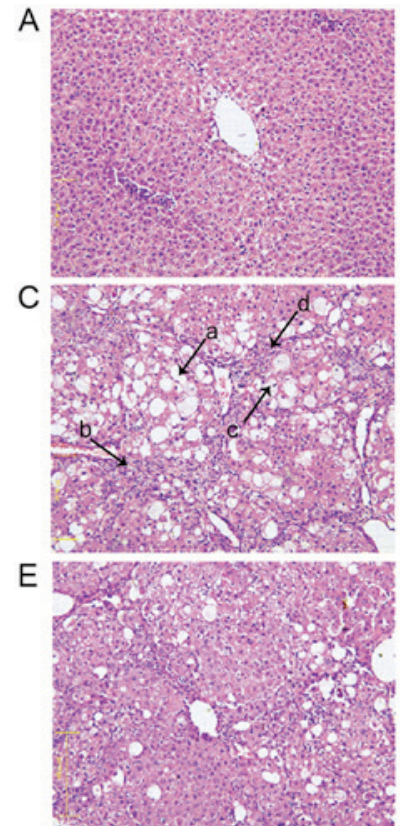

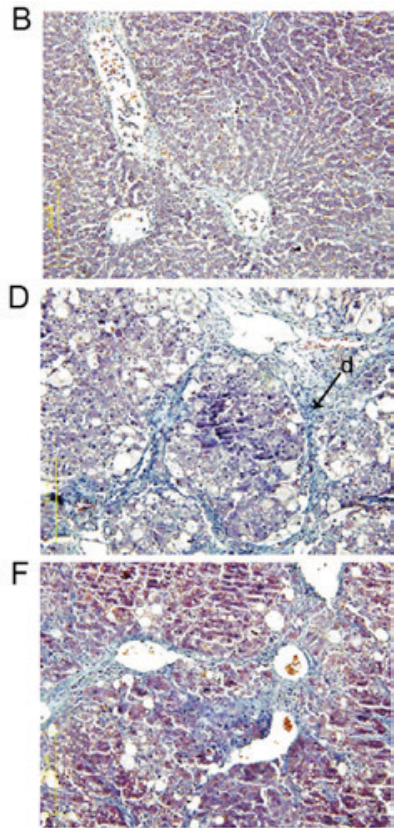

Figure 1. Histological examination of carbon tetrachloride-induced liver fibrosis in rats at 12 weeks. Magnification, x200. (A) HE and (B) Masson staining of liver tissue from the control group. (C) HE and (D) Masson staining of liver tissue from the model group. (E) HE and (F) Masson staining of liver tissue from the SGJPF group. Arrows: a, increased fatty degeneration; $b$, inflammatory cell infiltration; $c$, a dissolved nucleus; $d$, increased hyperplastic collagen fibers. HE, hematoxylin and eosin; SGJPF, Shu Gan Jian Pi formula.

Identification of endogenous metabolites. To select candidate biomarkers among the thousands of variables, variable importance projection (VIP) was applied. Only those biomarkers with a VIP $>1$ were considered (25). The remaining variables were subsequently assessed by ANOVA $(\mathrm{P}<0.05)$. To further identify potential biomarkers, commercial databases, including the KEGG (http://www.kegg.jp/kegg/) and PubChem Compound (https://pubchem.ncbi.nlm.nih.gov/), were used to search for metabolites. Based on these analyses, significantly altered serum metabolites between the groups included isoleucine, L-malic acid, D-erythro-sphingosine, putrescine, malonic acid, 3,6-anhydro-D-galactose, $\alpha$-ketoglutaric acid, ornithine, glucose, hippuric acid, tetrahydrocorticosterone and fucose (Table I). To improve the visualization of changes in the potential biomarkers, box-whisker plots were created using straight-forward statistical graphics to describe the distribution of several sets of quantitative data and compare their differences (26). The height of the box represents the interquartile range, the horizontal line represents the median, and the extensions up and down at the ends of the box represent the maximum and the minimum. As presented in Fig. 6, the 12 potential biomarkers exhibited differences between the control and model groups. SGJPF biomarker levels were comparable to those of the control group for certain metabolites.

Biological pathway and network analysis. To analyze the potential biomarkers involved in metabolic pathways, physiology, biochemistry and pathology knowledge was applied using CytoKEGG (Cytoscape 3.0.1 software; http://www. cytoscape.org/) analysis, which provided quantitative and metabolic information on organism metabolites. The present

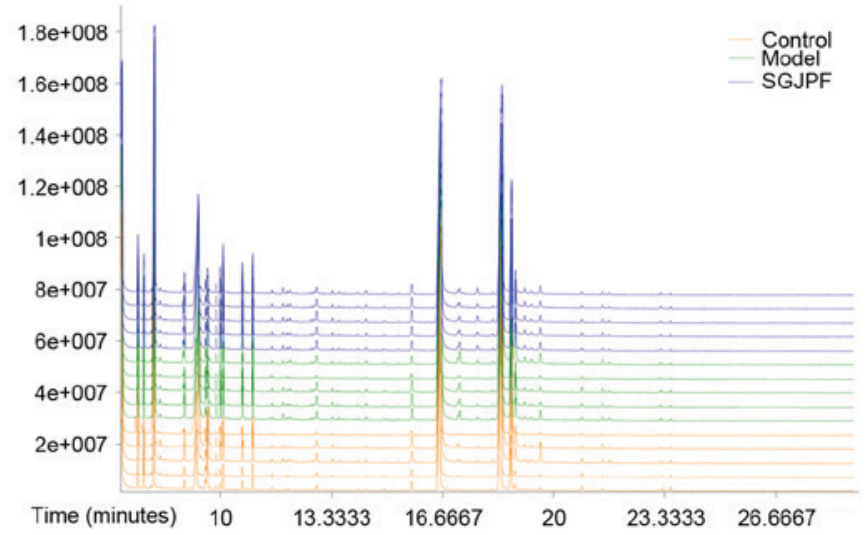

Figure 2. Typical gas chromatography-time of flight mass spectrometry total ion current chromatograms of rat serum samples at 12 weeks obtained from control, model and SGJPF groups. SGJPF, Shu Gan Jian Pi formula.

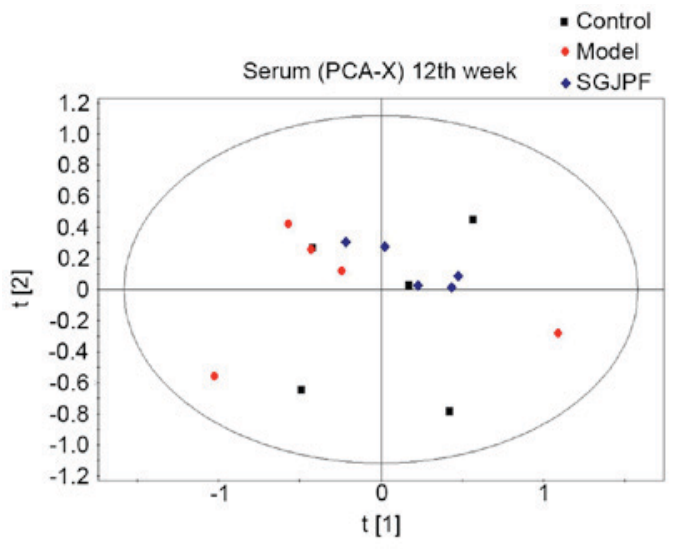

$R 2 X[1]=0.573752$ R2X [2] $=0.285619$ ellipse: hotelling $T 2(0.95)$

Figure 3. PCA score plot of carbon tetrachloride-induced liver fibrosis in rat serum samples obtained from the control (black bullet), model (red bullet) and SGJPF groups (blue bullet) at 12 weeks. PCA, principal component analysis; SGJPF, Shu Gan Jian Pi formula.

study determined that the metabolites were primarily involved in energy, amino acid, sphingolipid, cytochrome P450, glucose and water-electrolyte metabolism. The pathways were used to construct a potential biomarker metabolic pathway network (Fig. 7).

\section{Discussion}

Liver fibrosis, which is a pathological outcome of the wound-healing response of the liver to repeated injury by various damaging stimuli, is associated with an increased risk of liver cancer and liver failure. Liver cancer and liver failure are concerning due to their high morbidity and mortality rates $(27,28)$. The $\mathrm{CCl}_{4}$-induced animal model of liver fibrosis is commonly used due to the high success rate in this model; in addition, the model is easy to replicate (29). The resulting models are commonly successful, highly stable and time-saving when compared with ethanol and arsenic-induced liver fibrosis. This model induces pathological alterations similar to those observed during the development of human chronic hepatitis and hepatic fibrosis. Therefore, the modeling method has been widely used in screening anti-hepatic fibrosis drugs. The 
A

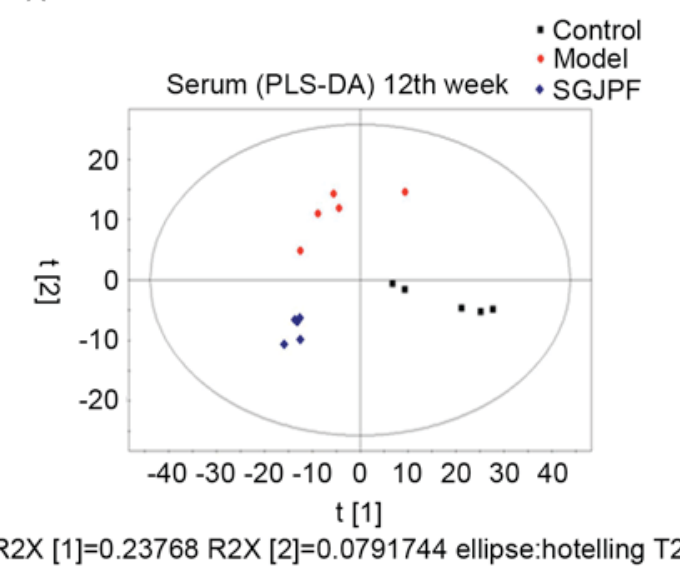

B

Serum. M57 (PLS-DA): validate model

\$ M57. DA1 intercepts: $R 2=(0.0,0.725)$, $\mathrm{Q} 2=(0.0,-0.22)$

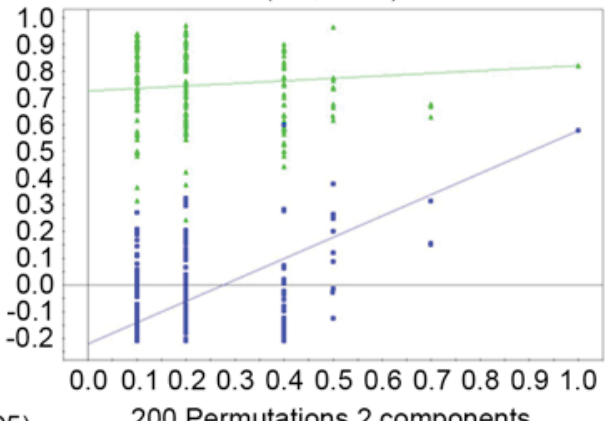

$\cdot \mathrm{R} 2$

$\cdot \mathrm{Q} 2$

Figure 4. PLS-DA model score plot and permutation test plot. (A) Score plot and (B) plot of the permutation test (200 times) of the PLS-DA model obtained from the control, model and SGJPF groups. A total of 200 permutations were performed and plotted according to the resulting $\mathrm{R}^{2}$ and $\mathrm{Q}^{2}$ values. Green triangle, $\mathrm{R}^{2}$; blue square, $\mathrm{Q}^{2} . \mathrm{R}^{2}=0.725$ and $\mathrm{Q}^{2}=-0.22$. PLS-DA, partial least squares-discriminate analysis; SGJPF, Shu Gan Jian Pi formula.

A

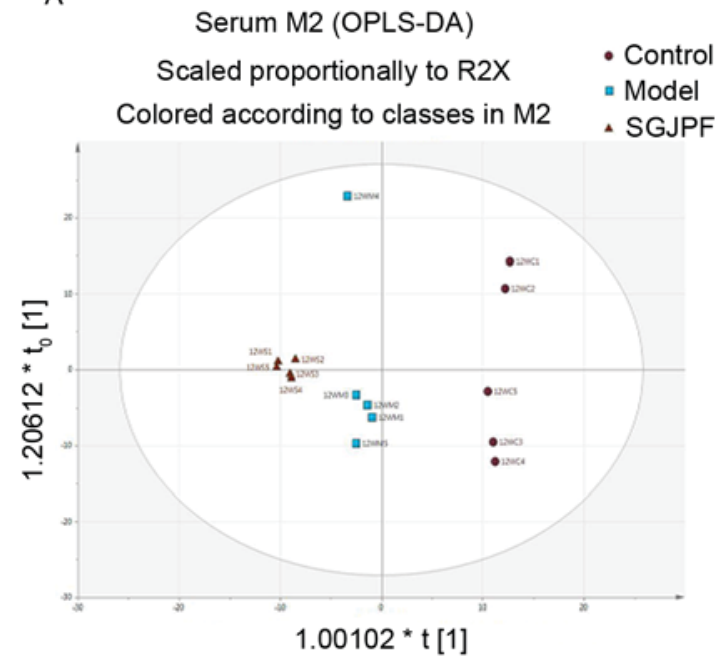

B

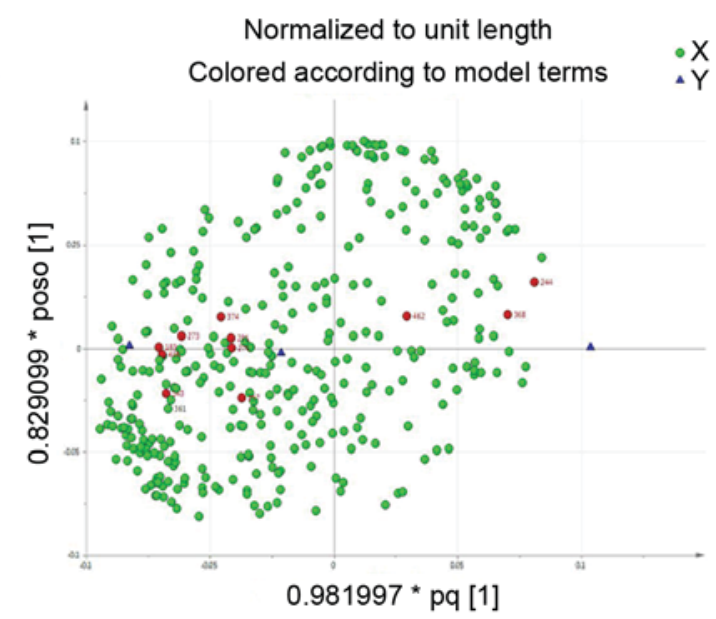

Figure 5. OPLS-DA analysis. (A) OPLS-DA score plot and (B) loading plot of rat serum samples obtained from the control, model and SGJPF groups. Each dot in the loading plot represents a metabolite that was identified as one of the most influential variables according to their respective contributions to the discrimination, red dots indicate potential biomarkers. OPLS-DA, orthogonal projections to latent structures-discriminate analysis; SGJPF, Shu Gan Jian Pi formula.

present study detected liver cell swelling, necrosis and notable collagen fiber deposition in rats with $\mathrm{CCl}_{4}$-induced fibrosis. SGJPF intervention led to improved liver tissue pathological damage and reduced liver fibrosis, indicating that it may protect against $\mathrm{CCl}_{4}$-induced liver fibrosis.

Metabonomics, named due to its combination of genomics and proteomics, is a research method using biological systems, which allows complex compound mixtures, such as those from TCM, to be holistically analyzed. Therefore, metabolomics is a promising approach to investigate TCM mechanisms and the associations between changes in potential metabolites and physiological systems (30). Serum sample analysis by GC-TOF/MS indicated that $\mathrm{CCl}_{4}$ exposure for 12 weeks induced metabolic alterations involving several associated metabolic pathways, including energy, amino acid, sphingolipid, glucose, cytochrome P450 and water-electrolyte metabolism. Liver fibrosis causes disturbances in the tricarboxylic acid (TCA) cycle, and also increases protein degradation from cell necrosis. When the liver is injured, the amino acid metabolic enzymes may be damaged and the amino acid metabolic control pathway deteriorates, causing an increase in serum amino acids (31). Furthermore, when protein synthesis in the liver is disturbed, necrobiosis and plasmatorrhexis processes release amino acids from hepatic cells into the bloodstream (32). Therefore, amino acid absorption into the liver to synthesize proteins is reduced. In addition, due to poor absorption of lipids, proteins and glucose by the liver, amino acids are generated by the decomposition of endogenous proteins for energy metabolism, which subsequently causes an increase in serum amino acids (33).

Ornithine is an amino acid that may be generated from glutamic acid or produced in the urea cycle by the hydrolysis of urea from arginine (34). Glutamic acid is one of the 20 proteinogenic amino acids and a key molecule in cellular metabolism. When the liver is injured, necrobiosis and plasmatorrhexis release glutamic acid from hepatic cells into the blood, leading to an increase in ornithine. Ornithine levels have 
Table I. Alterations in the levels of biomarkers in rat serum at 12 weeks in the control, model and SGJPF groups.

\begin{tabular}{|c|c|c|c|c|c|c|}
\hline No. & VarID & Compound name & RT (min) & VIP & Model group & SGJPF group \\
\hline 1 & 143 & Isoleucine & 9.87 & 1.78 & $\uparrow^{\mathrm{a}}$ & down \\
\hline 2 & 183 & D-Erythro-sphingosine & 10.82 & 1.76 & $\uparrow^{b}$ & down \\
\hline 3 & 231 & L-Malic acid & 12.27 & 1.53 & $\uparrow^{b}$ & down \\
\hline 4 & 244 & Putrescine & 12.65 & 1.49 & $\downarrow^{\mathrm{a}}$ & up \\
\hline 5 & 257 & Malonic acid & 12.98 & 1.52 & $\uparrow^{\mathrm{a}}$ & down \\
\hline 6 & 273 & 3,6-Anhydro-D-galactose & 13.48 & 1.68 & $\uparrow^{b}$ & down \\
\hline 7 & 274 & $\alpha$-Ketoglutaric acid & 13.49 & 1.98 & $\uparrow^{a}$ & down $^{c}$ \\
\hline 8 & 361 & Ornithine & 17.18 & 2.04 & $\uparrow^{\mathrm{a}}$ & $\operatorname{down}^{\mathrm{c}}$ \\
\hline 9 & 368 & Glucose & 17.55 & 1.58 & $\downarrow^{\mathrm{a}}$ & $u_{p}^{c}$ \\
\hline 10 & 374 & Hippuric acid & 17.77 & 1.79 & $\uparrow^{\mathrm{a}}$ & down \\
\hline 11 & 408 & Tetrahydrocorticosterone & 19.69 & 1.93 & $\uparrow^{\mathrm{a}}$ & down \\
\hline 12 & 462 & Fucose & 26.98 & 1.48 & $\downarrow^{\mathrm{a}}$ & up $^{c}$ \\
\hline
\end{tabular}

$\uparrow$, relatively high in the model group vs. control group; $\downarrow$, relatively low in the model group vs. control group; up, relatively high in the SGJPF group vs. model group; down, relatively low in the SGJPF group vs. model group. ${ }^{\text {a }}<<0.01$ and ${ }^{\mathrm{b}} \mathrm{P}<0.05$ vs. control group; ${ }^{\mathrm{P}}<0.05$ vs. model group. VarID, serum screening of 469 peaks to the serial number of compound; RT, retention time of substance; VIP, variable importance projection; SGJPF, Shu Gan Jian Pi formula.

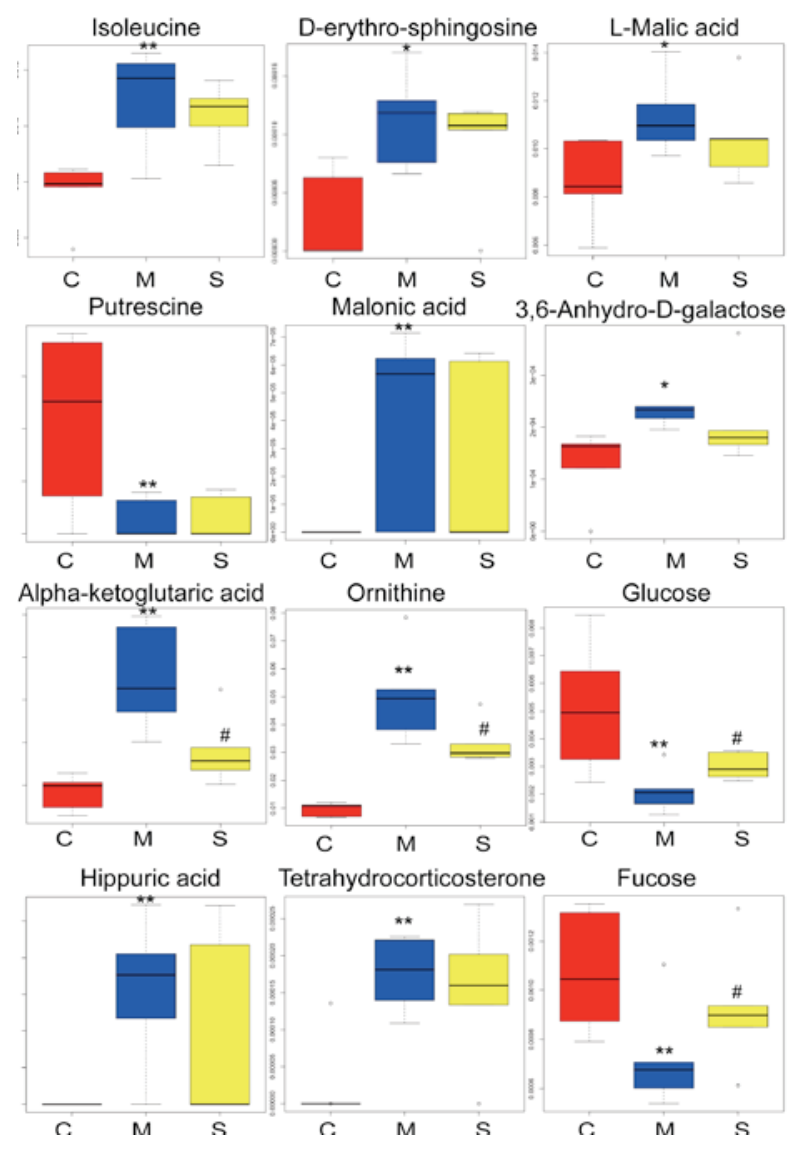

Figure 6. Box-whisker plots of serum isoleucine, D-erythro-sphingosine, L-malic acid, putrescine, malonic acid, 3,6-anhydro-D-galactose, $\alpha$-ketoglutaric acid, ornithine, glucose, hippuric acid, tetrahydrocorticosterone and fucose in $\mathrm{C}, \mathrm{M}$ and $\mathrm{S}$ groups. The height of the box represents the interquartile range, the horizontal line represents the median and the extensions up and down at the ends of the box represent the maximum and the minimum. C, control group; M, model group; S, Shu Gan Jian Pi formula group. ${ }^{*} \mathrm{P}<0.05$ and ${ }^{* *} \mathrm{P}<0.01$ vs. the control group; ${ }^{\#} \mathrm{P}<0.05$ and ${ }^{\# \#} \mathrm{P}<0.01$ vs. the model group. been previously reported to be higher in $\mathrm{CCl}_{4}$-treated rats (35), which is consistent with the results of the present study. The SGJPF group exhibited lower ornithine level compared with those of the model group, demonstrating that SGJPF treatment may improve the disordered amino acid metabolism observed in rats with $\mathrm{CCl}_{4}$-induced liver fibrosis.

Isoleucine is a branched-chain glucogenic amino acid, which is involved in the synthesis of liver glucose in mammals and participates in tricarboxylic acid cycling through acetyl CoA. It has previously been demonstrated that hepatopathy is associated with inflammation and oxidative stress (36). Under oxidative stress conditions, glycolysis and gluconeogenesis are inhibited. Therefore, it may be hypothesized that impaired gluconeogenesis may lead to elevated isoleucine in hepatic cells. In the present study isoleucine level were higher in the model group compared with the control group, indicating that abnormal amino acid metabolism follows $\mathrm{CCl}_{4}$ treatment. Although the level of glycine was not statistically significant when compared with the model group, the SGJPF group exhibited a downward trend, suggesting that SGJPF may affect the impaired amino acid metabolism to a certain degree in rats with $\mathrm{CCl}_{4}$-induced liver fibrosis.

Putrescine is a polyamine, which is related to the polyamine cadaverine (37). Both are produced by the breakdown of amino acids in living and dead organisms, and both are toxic in large doses (38). Putrescine attacks s-adenosyl methionine and converts it to spermidine. Spermidine subsequently attacks another s-adenosyl methionine, converting it to spermine. Putrescine is synthesized in small quantities by healthy living cells through the action of ornithine decarboxylase. The polyamines, of which putrescine is one of the simplest, appear to be growth factors necessary for cell division (39). They bind to polyamine regulatory sites of the N-methyl-D-aspartate (NMDA) receptor to generate NMDA. NMDA participates in the ornithine cycle, promoting oxygenand carbon dioxide-induced generation of urea and enhancing 


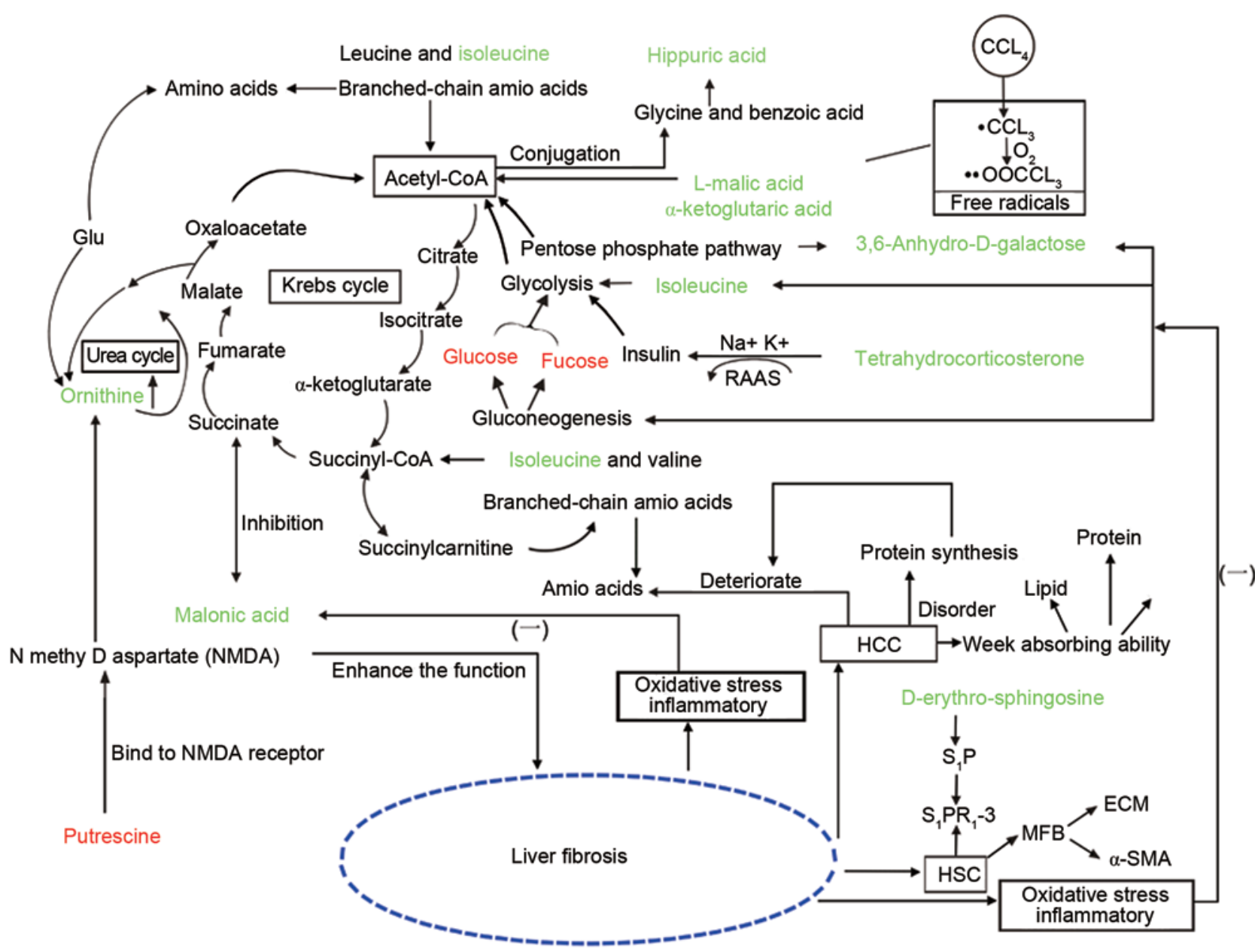

Figure 7. Global metabolomic network analysis of disturbed metabolic pathways in the control, model and SGJPF groups. Red and green words indicate that the metabolite was relatively low or relatively high in the model group vs. control group, respectively.

liver function. Therefore, in the medical industry, NMDA is primarily used as a liver function accelerant for the treatment of liver fibrosis $(40,41)$. These observations are consistent with the putrescine decrease observed in the model rat group in the present study, and this alteration may be associated with impaired amino acid metabolism. Following SGJPF treatment, the level of putrescine was marginally higher in the SGJPF group when compared with the model group, although it was not statistically significant, demonstrating that SGJPF may affect the impaired amino acid metabolism to a certain degree in rats with $\mathrm{CCl}_{4}$-induced liver fibrosis.

Sphingosine is an 18-carbon amino alcohol with a long unsaturated hydrocarbon chain. Its derivative, sphinganine, is the primary sphingolipid base in mammals. Sphingosine is phosphorylated in vivo via two kinases, sphingosine kinase type 1 and sphingosine kinase type 2 . This leads to the formation of sphingosine-1-phosphate (S1P), a potent signaling lipid. S1P primarily binds S1P receptors (S1PR) on the surface of the cell membrane (42). Hepatic stellate cells (HSCs) are persistently activated by liver fibrosis development (43). Previous research has demonstrated that S1PR1-3 may be expressed by HSCs $(44,45)$. HSCs transdifferentiate into myofibroblasts and synthesize ECM, which may have a role in the accelerated development of hepatic fibrosis. In addition, a previous study has demonstrated that S1P promoted fibroblast cell mitosis, stimulated fibroblast cell transdifferentiation into myofibroblasts, and induced $\alpha$-smooth muscle actin expression and ECM secretion (46). In the present study, D-erythro-sphingosine levels were higher in the model group compared with the control group. This alteration may be associated with the destruction of sphingolipid metabolism. The SGJPF group exhibited slightly lower D-erythro-sphingosine levels compared with the model group, indicating that treatment may restore impaired sphingolipid metabolism.

Following $\mathrm{CCl}_{4}$ injection in rats, $\mathrm{CCl}_{4}$ is decomposed into $\mathrm{CCl}_{3} \cdot$ and $\mathrm{CCl}_{3} \mathrm{O}_{3} \cdot$ free radicals, which induce an oxidative stress response by attacking liver cells. A previous study demonstrated that the TCA cycle is inhibited to reduce oxygen free radical generation under oxidative stress conditions, which is associated with liver diseases caused by various mechanisms (47). As important intermediate TCA products, malic acid and $\alpha$-ketoglutaric acid are energy supplies for the body. Malonic acid, whose structure is similar to succinic acid, competitively inhibits the production of fumaric acid, which controls the TCA cycle $(48,49)$. The present study observed that L-malic acid, malonic acid and $\alpha$-ketoglutaric acid levels were increased in the model group compared with the control group, potentially caused by disturbances in the TCA cycle. SGJPF treatment may affect the abnormal energy metabolism in rats with liver fibrosis. 
It has previously been indicated that liver diseases, such as liver fibrosis, are associated with disturbances in carbohydrate metabolism. In the process of liver disease, insulin inactivation is inhibited and serum insulin levels are distinctly elevated, which leads to increased glucose usage (50). Furthermore, fucose levels are increased, whereas glucose levels are significantly decreased. These observations are consistent with the decrease in saccharides observed in the model rat group compared with the control group in the present study. Saccharides, such as glucose, are the primary raw material that the liver uses to generate energy and other essential factors for the sugar metabolic pathways. 3,6-anhydro-D-galactose provides energy for the organism through the pentose phosphate and glycolysis pathways. The present study revealed that serum glucose and fucose levels were reduced, whereas serum 3,6-anhydro-D-galactose was increased in the model group compared with the control group. The SGJPF group exhibited higher glucose and fucose levels, and marginally lower 3,6-anhydro-D-galactose levels compared with the model group, which indicated that SGJPF may improve disordered glycometabolism in rats with liver fibrosis.

Compared with the control group, hippuric acid levels increased in the model group. Glycine is combined with benzoic acid to form hippuric acid under cytochrome P450 catalysis. Cytochrome P450 activity is associated with the development of liver fibrosis (51). The SGJPF group exhibited lower hippuric acid levels compared with those of the model group. This change indicated that SGJPF may improve impaired cytochrome P450 activity in rats with liver fibrosis. Tetrahydrocorticosterone is a corticoid, which includes glucocorticoid and mineralocorticoid. Aldosterone belongs to the family of mineralocorticoids, which maintain the balance of water-electrolyte metabolism, and is primarily regulated by the renin-angiotensin-aldosterone system (RAAS). Previous research indicated that RAAS exists locally in the liver. When hepatic fibrosis occurs, inactivated aldosterone decreases depending on how much the liver function is reduced (52). Therefore, the RAAS is associated with liver fibrosis formation. The alterations in the level of tetrahydrocorticosterone in the model group may be the result of water-electrolyte metabolism. The SGJPF group exhibited lower tetrahydrocorticosterone level compared with the model group. These changes indicated that SGJPF may improve impaired water-electrolyte metabolism in rats with liver fibrosis.

In conclusion, in the present study, $\mathrm{CCl}_{4}$ exposure induced liver damage and significantly altered several metabolic pathways. Histological results demonstrated that $\mathrm{CCl}_{4}$ treatment successfully induced liver fibrosis in rats. Pattern recognition with multivariate statistical analysis indicated that the metabolic profile of $\mathrm{CCl}_{4}$-induced liver fibrosis differed compared with the control group. A total of 12 serum metabolites, which are involved in energy, amino acid, sphingolipid, cytochrome P450, glucose and water-electrolyte metabolism, were identified as potential biomarkers. Following SGJPF intervention, the levels of these 12 serum metabolites tended to return to the normal level. The present study has identified novel targets for further research into the mechanisms of SGJPF and demonstrated that GC-TOFMS is a powerful technological platform for investigating drug mechanisms, particularly those of traditional Chinese compounds.

\section{Acknowledgements}

The present study was financially supported by the National Natural Science Foundation of China (grant no. 81102874) and the Leading Talents Introduction and Cultivation Plan Project of Colleges in Anhui province (grant no. gxfxZD2016118). The authors are grateful to Ms. Jie Xu and Dr Junliang Deng (Biotree Biotech Co., Ltd., Shanghai, China) for providing assistance with data analysis.

\section{References}

1. Kumar V, Mondal G, Dutta R and Mahato RI: Co-delivery of small molecule hedgehog inhibitor and miRNA for treating liver fibrosis. Biomaterials 76: 144-156, 2016

2. Bernuth S, Yagmur E, Schuppan D, Sprinzl MF, Zimmermann A, Schad A, Kittner JM, Weyer V, Knapstein J, Schattenberg JM, et al: Early changes in dynamic biomarkers of liver fibrosis in hepatitis $C$ virus-infected patients treated with sofosbuvir. Dig Liver Dis 48: 291-297, 2016.

3. Zhu C, Qi X, Li H, Peng Y, Dai J, Chen J, Xia C, Hou Y, Zhang W and Guo X: Correlation of serum liver fibrosis markers with severity of liver dysfunction in liver cirrhosis: A retrospective cross-sectional study. Int J Clin Exp Med 8: 5989-5998, 2015.

4. Gou X, Tao Q, Feng Q, Peng J, Zhao Y, Dai J, Wang W, Zhang Y, $\mathrm{Hu}$ Y and Liu P: Urine metabolic profile changes of CCl4-liver fibrosis in rats and intervention effects of Yi Guan Jian Decoction using metabonomic approach. BMC Complement Altern Med 13: 123, 2013.

5. Gao JR, Chen JF, Jiang H, et al: Effect of Shugan Jianpi Fang on Liver Metabonomics in Hepatic Fibrosis Rats Induced by Carbon Tetrachloride. J Traditional Chin Med 56: 961-966, 2015.

6. Cao CL, Yang SX, Zhang GL, et al: Curative effects of Ganle granule in treating chronic virus hepatitis of the liver-qi stagnation and the spleen deficiency type. Henan Traditional Chinese Medicine 27: 28-29, 2007.

7. Jiang H, Wu FR, Gao JR and Chen JF: Dynamic Study on Curative Effect of Shuganjianpifang Against Hepatic Fibrosis Induced by CCl4 in Rats. Zhong Yao Cai 37: 1815-1819, 2014 (In Chinese)

8. Zhang R, Chen JF, Gao JR, et al: Protective effect of Shuganjianpi Formula different extracts on chemical heptic injury. Pharmacology and Clinics of Chinese Materia Medica 30: 140-143, 2014.

9. Jiang H, Gao JR, Chen JF and Ji WB: Effect of Shuganjianpifang on the expression of BCL-2 and BAX in rats livers with hepatic fibrosis. Zhong Yao Cai 36: 776-780, 2013 (In Chinese).

10. Qin XJ, Gao JR, Jiang H, et al: Protective effects of ethanolic and aqueous extract of Shugan Jianpi Formula on CCl4-induced hepatic fibrosis in rats. Chinese Traditional Patent Medicine 37:1646-1651, 2015.

11. Wu H, Liu T, Ma C, Xue R, Deng C, Zeng H and Shen X: GC/MS-based metabolomic approach to validate the role of urinary sarcosine and target biomarkers for human prostate cancer by microwave-assisted derivatization. Anal Bioanal Chem 401: 635-646, 2011.

12. Chen J, Wang W, Lv S, Yin P, Zhao X, Lu X, Zhang F and Xu G: Metabonomics study of liver cancer based on ultra performance liquid chromatography coupled to mass spectrometry with HILIC and RPLC separations. Anal Chim Acta 650: 3-9, 2009.

13. Sun S, Dai J, Wang W, Cao H, Fang J, Hu YY, Su S and Zhang Y: Metabonomic Evaluation of ZHENG Differentiation and Treatment by Fuzhenghuayu Tablet in Hepatitis-B-Caused Cirrhosis. Evid Based Complement Alternat Med 2012: 453503, 2012.

14. Su ZH, Li SQ, Zou GA, Yu CY, Sun YG, Zhang HW, Gu Y and Zou ZM: Urinary metabonomics study of anti-depressive effect of Chaihu-Shu-Gan-San on an experimental model of depression induced by chronic variable stress in rats. J Pharm Biomed Anal 55: 533-539, 2011.

15. Gou X, Tao Q, Feng Q, Peng J, Sun S, Cao H, Zheng N, Zhang Y, $\mathrm{Hu} \mathrm{Y}$ and Liu P: Urinary metabonomics characterization of liver fibrosis induced by $\mathrm{CCl}_{4}$ in rats and intervention effects of Xia Yu Xue Decoction. J Pharm Biomed Anal 74: 62-65, 2013. 
16. Huang X, Shao L, Gong Y, Mao Y, Liu C, Qu H and Cheng Y: A metabonomic characterization of CCl4-induced acute liver failure using partial least square regression based on the GC/MS metabolic profiles of plasma in mice. J Chromatogr B Analyt Technol Biomed Life Sci 870: 178-185, 2008.

17. Gao S, Chen W, Peng Z, Li N, Su L, Lv D, Li L, Lin Q, Dong X, GuoZ and Lou Z: Urinary metabonomics elucidate the therapeutic mechanism of Orthosiphon stamineus in mouse crystal-induced kidney injury. J Ethnopharmacol 166: 323-332, 2015.

18. Hong Y, Han YQ, Wang YZ, Gao JR, Li YX, Liu Q and Xia LZ: Paridis Rhizoma Sapoinins attenuates liver fibrosis in rats by regulating the expression of RASAL1/ERK1/2 signal pathway. J Ethnopharmacol 192: 114-122, 2016.

19. Shannon P, Markiel A, Ozier O, Baliga NS, Wang JT, Ramage D, Amin N, Schwikowski B and Ideker T: Cytoscape: A software environment for integrated models of biomolecular interaction networks. Genome Res 13: 2498-2504, 2003.

20. Ding X, Liu R, Li W, Ni H, Liu Y, Wu D, Yang S, Liu J, Xiao B and Liu S: A metabonomic investigation on the biochemical perturbation in post-stroke patients with depressive disorder (PSD). Metab Brain Dis 31: 279-287, 2016.

21. Lin B, Zhang H, Lin Z, Fang Y, Tian L, Yang H, Yan J, Liu H, Zhang W and Xi Z: Studies of single-walled carbon nanotubes-induced hepatotoxicity by NMR-based metabonomics of rat blood plasma and liver extracts. Nanoscale Res Lett 8: 236 , 2013.

22. Szymanska E, Saccenti E, Smilde AK and Westerhuis JA Double-check: Validation of diagnostic statistics for PLS-DA models in metabolomics studies. Metabolomics 8 (Suppl 1): S3-S16, 2012.

23. Yang J, Zhao X, Liu X, Wang C, Gao P, Wang J, Li L, Gu J, Yang S and $\mathrm{Xu}$ G: High performance liquid chromatography-mass spectrometry for metabonomics: Potential biomarkers for acute deterioration of liver function in chronic hepatitis B. J Proteome Res 5: 554-561, 2006.

24. Ma J, Yu J, Su X, Zhu C, Yang X, Sun H, Chen D, Wang Y, Cao H and Lu J: UPLC-MS-based serum metabonomics for identifying acute liver injury biomarkers in Chinese miniature pigs. Toxicol Lett 225: 358-366, 2014

25. Wang L, Hou E, Wang L, Wang Y, Yang L, Zheng X, Xie G, Sun Q, Liang $M$ and Tian Z: Reconstruction and analysis of correlation networks based on GC-MS metabolomics data for young hypertensive men. Anal Chim Acta 854: 95-105, 2015.

26. Blydt-Hansen TD, Sharma A, Gibson IW, Mandal R and Wishart DS: Urinary metabolomics for noninvasive detection of borderline and acute $\mathrm{T}$ cell-mediated rejection in children after kidney transplantation. Am J Transplant 14: 2339-2349, 2014

27. Barr RG, Ferraioli G, Palmeri ML, Goodman ZD, Garcia-Tsao G Rubin J, Garra B, Myers RP, Wilson SR, Rubens D and Levine D: Elastography assessment of liver fibrosis: Society of radiologists in ultrasound consensus conference statement. Radiology 276 : 845-861, 2015.

28. Barta A, Janega P, Babal P, Murár E, Cebova M and Pechánová O: The effect of curcumin on liver fibrosis in the rat model of microsurgical cholestasis. Food Funct 6: 2187-2193, 2015.

29. Jin S, Cao H, Wang K, Li Y and Bai B: Preventative effects of prostaglandin E1 in combination with iodized olive oil on liver fibrosis after transcatheter arterial chemoembolization in a rabbit model of CCl4-induced liver fibrosis. Can J Physiol Pharmacol 93: 451-457, 2015

30. Sun H, Zhang AH, Zou DX, Sun WJ, Wu XH and Wang XJ: Metabolomics coupled with pattern recognition and pathway analysis on potential biomarkers in liver injury and hepatoprotective effects of yinchenhao. Appl Biochem Biotechnol 173 857-869, 2014.

31. Zeng J, Yin P, Tan Y, Dong L, Hu C, Huang Q, Lu X, Wang H and Xu G: Metabolomics study of hepatocellular carcinoma: Discovery and validation of serum potential biomarkers by using capillary electrophoresis-mass spectrometry. J Proteome Res 13: 3420-3431, 2014

32. Pan L, Qiu Y, Chen T, Lin J, Chi Y, Su M, Zhao A and Jia W: An optimized procedure for metabonomic analysis of rat liver tissue using gas chromatography/time-of-flight mass spectrometry. J Pharm Biomed Anal 52: 589-596, 2010.
33. Yu K, Sheng G, Sheng J, Chen Y, Xu W, Liu X, Cao H, Qu H, Cheng Y and Li L: A metabonomic investigation on the biochemical perturbation in liver failure patients caused by hepatitis B virus. J Proteome Res 6: 2413-2419, 2007.

34. Silva MA, Mirza DF, Buckels JA, Bramhall SR, Mayer D, Wigmore SJ, Murphy N and Richards DA: Arginine and urea metabolism in the liver graft: A study using microdialysis in human orthotopic liver transplantation. Transplantation 82: 1304-1311, 2006

35. Chen $\mathrm{B}$ and He S: The role of glutamine and arginine in rat hepatic fibrosis. Chine J General Sur 17: 883-886, 2008

36. Ding Y, Rao SX, Zhu T, Chen CZ, Li RC and Zeng MS: Liver fibrosis staging using T1 mapping on gadoxetic acid-enhanced MRI compared with DW imaging. Clin Radiol 70: 1096-1103, 2015.

37. Bagheryan Z, Noori A, Zahra Bathaie S, Yousef-Elahi M and Mousavi MF: Preparation of a new nanobiosensor for the determination of some biogenic polyamines and investigation of their interaction with DNA. Biosens Bioelectron 77: 767-773, 2016

38. De Sarro GB, Bagetta G, Spagnolo C and Nisticò G: Antagonists of N-methyl-D-aspartate receptors block seizures induced by putrescine in the deep prepiriform cortex. Neuropharmacology 32 : 43-50, 1993.

39. Paschalidis KA and Roubelakis-Angelakis KA: Sites and regulation of polyamine catabolism in the tobacco plant. Correlations with cell division/expansion, cell cycle progression, and vascular development. Plant Physiol 138: 2174-2184, 2005.

40. Ota H, Ogawa S, Ouchi Y and Akishita M: Protective effects of NMDA receptor antagonist, memantine, against, senescence of PC12 cells: A possible role of nNOS and combined effects with donepezil. Exp Gerontol 72: 109-116, 2015.

41. Choudhury S and Borah A: Activation of NMDA receptor by elevated homocysteine in chronic liver disease contributes to encephalopathy. Med Hypotheses 85: 64-67, 2015.

42. Aarthi JJ, Darendeliler MA and Pushparaj PN: Dissecting the role of the S1P/S1PR axis in health and disease. J Dent Res 90: 841-854, 2011.

43. Zhang F, Lu C, Xu W, Shao J, Wu L, Lu Y and Zheng S: Curcumin raises lipid content by Wnt pathway in hepatic stellate cell. J Surg Res 200: 460-466, 2016

44. Yang L, Yue S, Yang L, Liu X, Han Z, Zhang Y and Li L: Sphingosine kinase/sphingosine 1-phosphate (S1P)/S1P receptor axis is involved in liver fibrosis-associated angiogenesis. J Hepatol 59: 114-123, 2013

45. Li C, Zheng S, You H, Liu X, Lin M, Yang L and Li L: Sphingosine 1-phosphate (S1P)/S1P receptors are involved in human liver fibrosis by action on hepatic myofibroblasts motility. J Hepatol 54: 1205-1213, 2011.

46. Sobel K, Menyhart K, Killer N, Renault B, Bauer Y, Studer R, Steiner B, Bolli MH, Nayler O and Gatfield J: Sphingosine 1-phosphate $(\mathrm{S} 1 \mathrm{P})$ receptor agonists mediate pro-fibrotic responses in normal human lung fibroblasts via S1P2 and S1P3 receptors and Smad-independent signaling. J Biol Chem 288: 14839-14851, 2013.

47. Srinivasan PK, Yagi S, Nagai K, Afify M, Hata K, Uemoto S and Tolba RH: Impact of oxygen free radicals in rat partial liver transplantation. J Surg Res 191: 469-475, 2014.

48. Peti-Peterdi J: Mitochondrial TCA cycle intermediates regulate body fluid and acid-base balance. J Clin Invest 123: 2788-2790, 2013.

49. Wang D, Zhang P, Wang X, Wang Y, Zhou Z and Zhu W: NMRand LC-MS/MS-based urine metabolomic investigation of the subacute effects of hexabromocyclododecane in mice. Environ Sci Pollut Res Int 23: 8500-8507, 2016.

50. Grancini V, Trombetta M, Lunati ME, Zimbalatti D, Boselli ML, Gatti S, Donato MF, Resi V, D'Ambrosio R, Aghemo A, et al: Contribution of $\beta$-cell dysfunction and insulin resistance to cirrhosis-associated diabetes: Role of severity of liver disease. J Hepatol 63: 1484-1490, 2015.

51. Chiu KW, Nakano T, Chen KD, Hsu LW, Lai CY, Huang CY, Cheng YF, Goto S and Chen CL: Cytochrome P450 in living donor liver transplantation. J Biomed Sci 22: 32, 2015.

52. Yoshiji H, Noguchi R and Fukui H: RAAS inhibitors as therapeutic alternatives in the treatment of chronic liver diseases. Nihon Rinsho 67: 799-806, 2009 (In Japanese). 